\title{
In situ optical spectroscopy of Ga dimers on GaP, GaAs, and GaSb by surface chemical modulation
}

\author{
Pablo Aitor Postigo, * Gaspar Armelles, Thomas Utzmeier, and Fernando Briones \\ Instituto de Microelectrónica de Madrid (CSIC), Isaac Newton, 8 Parque Tecnológico de Madrid, 28760 Tres Cantos, Madrid, Spain
}

(Received 21 May 1997)

\begin{abstract}
The optical characterization of the surface anisotropy of three Ga-based semiconductor compounds using an in situ technique based on the chemical modulation of the surface is described. In this technique, the anisotropic optical reflectivity is modulated by a periodic variation of the surface stoichiometry using valved pulsed cells for group-V elements (As, $\mathrm{P}, \mathrm{Sb}$ ). The substrate is maintained at sufficiently high temperature in order to obtain rapid desorption of group-V molecules from the surface during flux interruptions. Linearly polarized light, reflected at near normal incidence by the sample, is collected separately along one of the two principal axes of the crystal, [110] and [1 $\overline{1} 0]$. The change in the surface coverage induces a change in the intensity of the reflected light, and the normalized variation $\Delta R / R$ is recorded as a function of wavelength. Spectra for (001) surfaces in the 1-3 eV range have been obtained with this method for a set of Ga-based binary III-V compounds GaP, GaAs, and GaSb, showing well-defined features for light polarized along the [110] direction, parallel to Ga dimers. These observed maxima are attributed to transitions between the occupied Ga dimer and the unoccupied dangling bond bands, at characteristic energies in each material. [S0163-1829(97)00536-5]
\end{abstract}

\section{INTRODUCTION}

Optical reflection has been widely used to the in situ study of semiconductor surfaces and to monitor surface structures during epitaxial growth. Previous work in surface optical analysis has been done using polarization-modulated techniques. ${ }^{1}$ Reflectance difference spectroscopy (RDS) (Ref. 2) and surface photoabsorption (SPA) (Ref. 3) are the two most commonly used optical techniques to provide information on dielectric responses and growth mechanisms of surfaces and interfaces. RDS is used to measure the difference between normal-incidence reflectances for light polarized along the two principal axes in the surface. On the other hand, SPA measures the change in reflectance caused by surface modification using $p$-polarized light impinging on the surface at the angle of incidence close to the pseudoBrewster angle of the substrate. A great variety of semiconductor surfaces such as $\mathrm{Si}^{4}{ }^{4} \mathrm{Ge},{ }^{5} \mathrm{GaAs},{ }^{6-8} \mathrm{InAs},{ }^{9} \mathrm{InGaAs},{ }^{10}$ or AlAs (Ref. 11) grown by molecular beam epitaxy (MBE) or metal organic chemical vapor deposition (MOCVD) have been studied in situ by means of these methods, providing useful information about the surface structure and growth processes of such materials.

We present a technique for the in situ study of the surfaces of III-V compounds based on the chemical modulation of the surface coverage. This technique has been applied to the measurement of the optical surface anisotropy of three Ga-based compounds grown by MBE at two crystallographic directions [110] and [110] and it seems valid for the surface characterization of all III-V compounds, showing optical features that, to our knowledge, have not been observed, except for GaAs, until now.

\section{EXPERIMENTAL}

Light from a halogen lamp was passed through a heatable viewport used for pyrometric measurements and directed onto the substrate at near normal incidence. A heated viewport $\left(T>450{ }^{\circ} \mathrm{C}\right)$ was used to avoid deposition of the group- $\mathrm{V}$ element during measurements. Reflected light was then passed through a polarizer whose transmission axis was parallel to one of the principal axes of the substrate, which was at an angle of $45^{\circ}$ from the horizontal axis. This reflected light was focused by means of a lens and collected by a Si or GaInAs $p-i-n$ photodiode mounted on the exit slit of a $0.22 \mathrm{~m}$ focal-length monochromator. The change in optical reflectivity with changing surface coverage during deposition and posterior desorption of the group $\mathrm{V}$ element molecules was $\mathrm{dc}$ amplified and recorded by computer. GaP and GaAs epitaxial buffer layers were grown on (001) oriented GaP and GaAs substrates, respectively, by MBE. GaSb was also grown on (001) GaAs substrate with sufficient thickness ( $\sim 1$ $\mu \mathrm{m})$ to obtain a flat morphology and relaxed material. Pulses of $\mathrm{As}_{4}, \mathrm{P}_{2}$, and $\mathrm{Sb}_{4}$ were obtained using valved pulsed cells and solid sources. The substrate temperature was high enough in each case as to permit rapid desorption $\left(t_{d}<3 s\right)$ of group- $\mathrm{V}$ element molecules from the surface. The corresponding surface reconstruction changes were monitored by reflection high-energy electron diffraction (RHEED). Figure 1 shows $\Delta R / R$ versus time for $\mathrm{As}_{4}$ pulses over GaAs for different wavelengths and with the polarizer parallel to the [110] direction. The beam equivalent pressure (BEP) of $\mathrm{As}_{4}$ is $4 \times 10^{-6}$ Torr and the pulse length is $0.3 \mathrm{~s}$, with a constant substrate temperature $T_{s}=556^{\circ} \mathrm{C}$. The initial stage (As off) RHEED pattern corresponds to a $(4 \times 2)$ Ga-stabilized surface, and the initial level of reflected light intensity is arbitrarily fixed as $R_{\mathrm{Ga}}$. When the $\mathrm{As}_{4}$ cell is open (As on), the intensity of reflected light decreases to reach $R_{\mathrm{As}}$, where surface saturation in As occurs. A clear RHEED change is observed, showing a $(2 \times 4)$ diffraction pattern at this moment. After that, the $\mathrm{As}_{4}$ beam is interrupted (As off) and desorption of As from the surface occurs. The intensity of light increases exponentially with time until the initial level of 


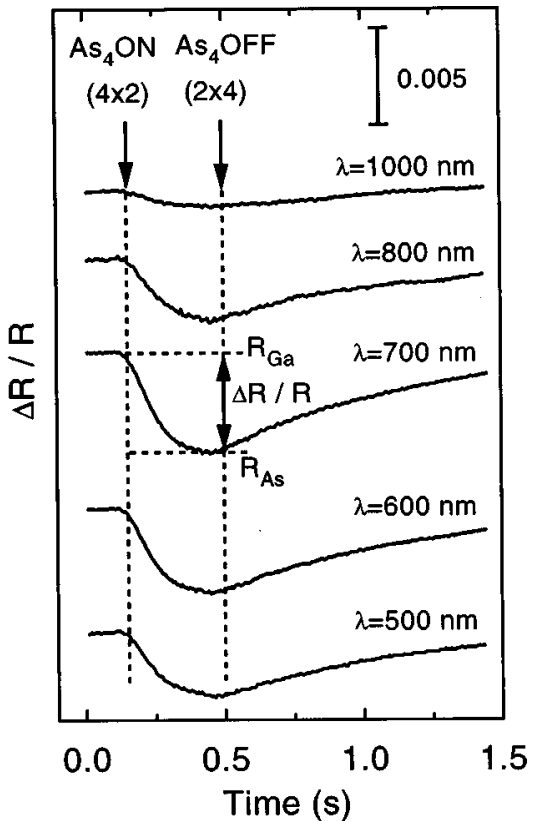

FIG. 1. Time evolution of [110] polarization reflectivity of $\mathrm{GaAs}$ during and after each $\mathrm{As}_{4}$ pulse for various wavelengths. The pulse length is $0.3 \mathrm{~s}$, and the normalized change in the reflectivity is marked as $\Delta R / R=\left(R_{\mathrm{Ga}}-R_{\mathrm{As}}\right) / R_{\mathrm{Ga}}$. The substrate temperature $\left(T_{s}=556{ }^{\circ} \mathrm{C}\right.$ ) needs to be sufficiently high to quickly achieve a fully desorbed $(4 \times 2)$ surface state after $\mathrm{As}_{4}$ is switched off.

intensity is recovered, and a RHEED $(4 \times 2)$ pattern is observed again. We define the increment in the reflectivity caused by the change in the coverage $\Delta R$ as $\Delta R=R_{\mathrm{Ga}}{ }^{-} R_{\mathrm{As}}$ and its normalized value, $\Delta R / R=\left(R_{\mathrm{Ga}}-R_{\mathrm{As}}\right) / R_{\mathrm{Ga}}$. The maximum $\Delta R / R$ value is $\cong \frac{1}{500}$ and since the recorded signal is quite noisy, the deposition-desorption process is repeated for each wavelength (about 20 times is sufficient) and the signal averaged until mean quadratic standard deviation of $\Delta$ $R$ is lower than a fixed value (usually $5 \%$ ). $\Delta R / R$ is recorded in that way as a function of wavelength in the $1-3 \mathrm{eV}(400-$ $1200 \mathrm{~nm})$ range for both selected polarizer azimuths [110] and $[1 \overline{1} 0]$.

\section{RESULTS}

Figure 2 shows the spectra measured for $\mathrm{GaP}, \mathrm{GaAs}$, and $\mathrm{GaSb}$. The substrate temperatures used for measurements were $T_{s}=598{ }^{\circ} \mathrm{C}$ for $\mathrm{GaP}, 556{ }^{\circ} \mathrm{C}$ for $\mathrm{GaAs}$, and $462^{\circ} \mathrm{C}$ for $\mathrm{GaSb}$, where a rapid desorption of group- $\mathrm{V}$ molecules from the surface was observed by RHEED, showing the change in the surface reconstruction from $(2 \times 4)$ to $(2 \times 2)$ in GaP, $(4$ $\times 2)$ to $(2 \times 4)$ in $\mathrm{GaAs}$, and $(1 \times 3)$ to $c(2 \times 6)$ in $\mathrm{GaSb}$ when the group-V element cell is open. Pulses with a BEP amplitude of $2.5 \times 10^{-6}$ Torr for $\mathrm{P}_{2}$ in GaP, $4 \times 10^{-6}$ Torr for $\mathrm{As}_{4}$ in GaAs, and $1.7 \times 10^{-6}$ Torr for $\mathrm{Sb}_{4}$ in $\mathrm{GaSb}$ were used, with pulse lengths of $0.5,0.3$, and $0.5 \mathrm{~s}$, respectively. After the group- $\mathrm{V}$ element cell is shutdown, desorption occurs during a time $t_{d}=2.5 \mathrm{~s}$ for $\mathrm{GaP}, 2.2 \mathrm{~s}$ for GaAs, and $2.5 \mathrm{~s}$ for $\mathrm{GaSb}$.

Well-defined maxima appear in the [110] direction, parallel to $\mathrm{Ga}$ dimers. These maxima are located at $\sim 2.2 \mathrm{eV}$ (570 $\mathrm{nm})$ for $\mathrm{GaP}, \sim 1.8 \mathrm{eV}(700 \mathrm{~nm})$ for $\mathrm{GaAs}$, and $\sim 1.2 \mathrm{eV}$ $(1050 \mathrm{~nm})$ for $\mathrm{GaSb}$. In the case of GaAs, the energy posi-

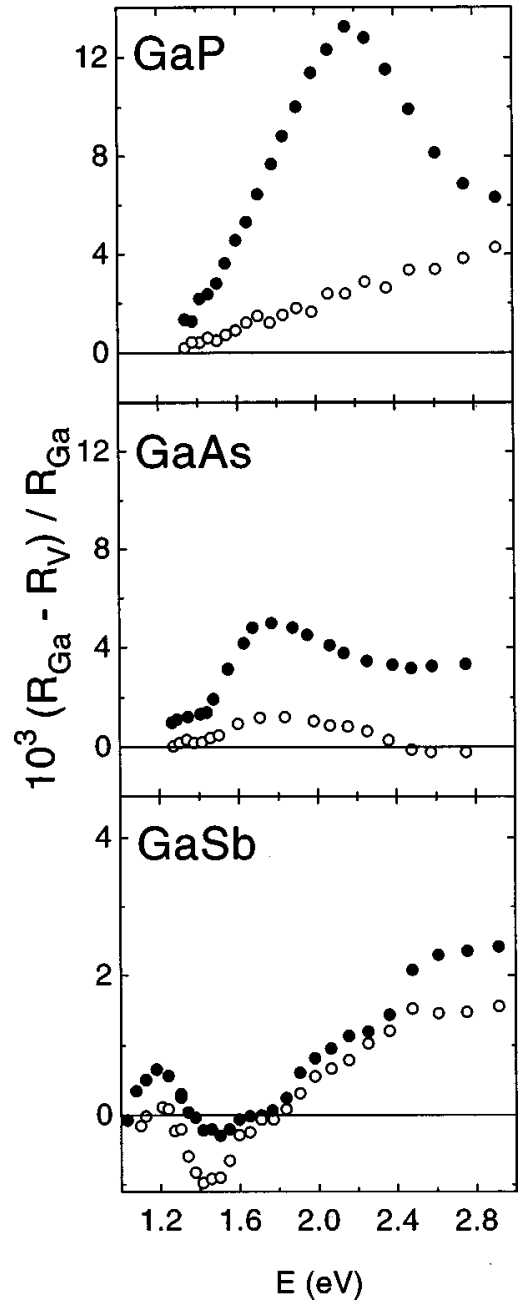

FIG. 2. Spectra for GaP, GaAs, and GaSb. Solid dots are for [110] polarization whereas open dots are for [110]. Substrate temperatures during measurement were $T_{s}=598{ }^{\circ} \mathrm{C}$ for $\mathrm{GaP}, 556{ }^{\circ} \mathrm{C}$ for $\mathrm{GaAs}$, and $462{ }^{\circ} \mathrm{C}$ for $\mathrm{GaSb}$.

tion agrees well with that observed by Aspnes et al. ${ }^{12}$ at 1.8 $\mathrm{eV}$ and assigned to an optical transition between the occupied Ga-dimer and unoccupied dangling-bond bands. ${ }^{13}$ Our technique establishes the difference of reflectivity between two surfaces, covered by group-III and group-V elements, respectively. In this polarization, and in the measured spectral range, surfaces terminated in group- $\mathrm{V}$ element are not expected to have abrupt optical features. ${ }^{13}$ By means of the expressions that yield the intensity of the reflected light by a thin film ${ }^{14}$ and according to the behavior of the dielectric function of the bulk material by Aspnes et al. ${ }^{15}$ we obtain that $\Delta R / R$ is mainly related with the imaginary part of the surface dielectric function that represents the surface absorption. For these reasons, we assign the maxima to be due to transitions between occupied Ga-dimer bands and unoccupied dangling-bond bands at characteristic energies in each material. Moreover, a simple calculation based on a tightbinding (TB) model with interactions up to the first neighbors and an $s p^{3} s^{*}$ basis, for a schematic Ga-dimer molecule with a geometry similar to the atomic surface arrangement, shows the same observed tendency (3.09 eV for GaP, 2.98 $\mathrm{eV}$ for $\mathrm{GaAs}$, and $2.49 \mathrm{eV}$ for $\mathrm{GaSb}$ ) for the shift in energy of the maximum with the material. The TB parameters for 
the bonds between $\mathrm{Ga}$ and $\mathrm{P}, \mathrm{As}$, and $\mathrm{Sb}$, were taken from Ref. 16 and the Ga-dimer interaction parameters are taken from the As-dimer interaction parameters of Ref. 17 scaled according to Harrison's $1 / d^{2}$ rule. ${ }^{18}$ In this calculation we have assumed that the Ga-Ga distance does not change from one compound to another, being twice the covalent radius of the atomic $\mathrm{Ga}$, whereas the distance between $\mathrm{Ga}$ and the group- $\mathrm{V}$ atom bonded changes from one compound to another, according to the lattice parameter. This calculation also explains the decrease in the intensity of the transition as the lattice parameter of the compound increases. The calculated energies for the transitions are higher than the experimental ones. Part of this discrepancy can be attributed to the use of TB parameters that correspond to a fit of the $0 \mathrm{~K}$ band structures whereas the experiment is done in the 700-800 K range. Also the effective $\mathrm{Ga}-\mathrm{Ga}$ distance could be lower than twice the covalent radius of $\mathrm{Ga}$, further reducing the transitions energies. In the [1 $\overline{10}]$ direction, that is, parallel to group- $\mathrm{V}$ dimers, the signal is much lower than in the [110] direction, and no clear deeps have been found except for $\mathrm{GaSb}$, at an energy of $\sim 1.4 \mathrm{eV}(850 \mathrm{~nm})$. This peak is assigned to a transition from the occupied lone-pair band to the unoccupied $\mathrm{Sb}$ dimer band. Probably another peak exists for GaAs near $2.7 \mathrm{eV}$, as has been observed previously, ${ }^{12}$ and at

*Present address: Massachusetts Institute of Technology, Department of Electrical Engineering, Room 13-3062, 77 Mass. Ave., Cambridge, MA 02139. Electronic address: aitor@oevlsi.mit.edu

${ }^{1}$ P. Chiaradia, A. Cricenti, G. Chiarotti, and S. Selci, Phys. Rev. Lett. 52, 13 (1987); 52, 1145 (1987).

${ }^{2}$ D. E. Aspnes, J. P. Harbison, A. A. Studna, and L. T. Florez, Phys. Rev. Lett. 59, 1687 (1987).

${ }^{3}$ N. Kobayashi and Y. Horikoshi, Jpn. J. Appl. Phys. 28, L1880 (1989).

${ }^{4}$ D. E. Aspnes and A. A. Studna, Phys. Rev. Lett. 54, 1956 (1985).

${ }^{5}$ T. Yasuda, L. Mantese, U. Rossow, and D. E. Aspnes, Phys. Rev. Lett. 74, 3431 (1995).

${ }^{6}$ I. Kamiya, D. E. Aspnes, L. T. Florez, and J. P. Harbison, Phys. Rev. B 46, 15894 (1992).

${ }^{7}$ K. Uwai, N. Kobayashi, Appl. Phys. Lett. 65, 150 (1994).

${ }^{8}$ J. Jönsson, K. Deppert, S. Jeppesen, G. Paulsson, L. Samuelson, and P. Schmidt, Appl. Phys. Lett. 56, 2414 (1990).

${ }^{9}$ R. Arès, C. A. Tran, and S. P. Watkins, Appl. Phys. Lett. 67, 1576 (1995). higher energies inside the uv range for $\mathrm{GaP}$, all related with these kinds of transitions.

In summary, we have applied an optical characterization technique based on the chemical modulation of the surface to obtain spectroscopic information on reconstruction associated surface states on III-V compounds. Well-defined features have been found for $\mathrm{GaP}, \mathrm{GaAs}$, and $\mathrm{GaSb}$ in the 1-3 $\mathrm{eV}$ range for light polarized along [110] direction, parallel to the Ga-Ga dimers. For the case of GaAs, the position of the peak agrees with previous measurements by conventional RDS. The shift in peak position with changing anion $(\mathrm{Ga} X$; $X=\mathrm{P}, \mathrm{As}, \mathrm{Sb})$ shows the strong influence of the group- $\mathrm{V}$ chemical bond on the absorption energy of the Ga-Ga dimer of materials. This has been assigned to a transition between bonding Ga-dimer orbitals and empty lone-pair states, and has been observed, as far as we know, for the first time in these compounds.

\section{ACKNOWLEDGMENTS}

One of the authors (P. A.) acknowledges the support received from the Basque Government. This work was partially supported by a MAT95-0966 Research Action.

${ }^{10}$ B. A. Philips, I. Kamiya, K. Hingerl, L. T. Florez, D. E. Aspnes, S. Mahajan, and J. P. Harbison, Phys. Rev. Lett. 74, 3640 (1995).

${ }^{11}$ M. Wassermeier, I. Kamiya, D. E. Aspnes, L. T. Florez, J. P. Harbison, and P. M. Petroff, J. Vac. Sci. Technol. B 9, 2263 (1991).

${ }^{12}$ D. E. Aspnes, Y. C. Chang, A. A. Studna, L. T. Florez, H. H. Farrell, and J. P. Harbison, Phys. Rev. Lett. 64, 192 (1990).

${ }^{13}$ Y. C. Chang and D. E. Aspnes, Phys. Rev. B 41, 12002 (1990).

${ }^{14}$ J. D. E. McIntyre and D. E. Aspnes, Surf. Sci. 24, 417 (1971).

${ }^{15}$ D. E. Aspnes and A. A. Studna, Phys. Rev. B 27, 985 (1983).

${ }^{16}$ P. K. Larsen, J. F. van der Veen, A. Mazur, J. Pollmann, J. H. Neave, and B. A. Joyce, Phys. Rev. B 26, 3222 (1982).

${ }^{17}$ P. Vogl, H. P. Hjalmarson, and J. D. Dow, J. Phys. Chem. Solids 44, 365 (1983).

${ }^{18}$ W. A. Harrison, Electronic Structure and the Properties of Solids (Freeman, San Francisco, 1980). 